\title{
Cell Type-Dependent Integrin Distribution in Adhesion and Migration Responses on Protein-Coated Microgrooved Substrates
} \author{
Adria Sales, ${ }^{*}, \dagger, \#$ Karen Ende, ${ }^{\ddagger}, \nabla$ Jennifer Diemer, ${ }^{\dagger}$ Adriana R. Kyvik, Jaume Veciana, ${ }^{\ddagger}{ }^{\ddagger}$ Imma Ratera, ${ }^{\ddagger \oplus 0}$
} Ralf Kemkemer, ${ }^{\dagger, \S}$ Joachim P. Spatz, ${ }^{\dagger, \|}$ and Judith Guasch*, ${ }^{*,+} \perp_{\odot}$

\begin{abstract}
${ }^{\dagger}$ Department of Cellular Biophysics, Max Planck Institute for Medical Research, Jahnstrasse 29, D-69120 Heidelberg, Germany
${ }^{\ddagger}$ Department of Molecular Nanoscience and Organic Materials, Institute of Materials Science of Barcelona (ICMAB-CSIC) and Networking Research Center on Bioengineering, Biomaterials and Nanomedicine (CIBER-BBN), Campus UAB, E-08193 Bellaterra, Spain

${ }^{\S}$ Reutlingen University, Alteburgstrasse 150, D-72762 Reutlingen, Germany

"Department of Biophysical Chemistry, University of Heidelberg, INF 253, D-69120 Heidelberg, Germany

${ }^{\perp}$ Dynamic Biomaterials for Cancer Immunotherapy, Max Planck Partner Group, ICMAB-CSIC, Campus UAB, E-08193 Bellaterra, Spain
\end{abstract}

\section{Supporting Information}

ABSTRACT: Although integrins are responsible for the interaction of cells with their environment, e.g., the extracellular matrix or artificial substrates, there is still a lack of knowledge about their role in cell adhesion and migration on protein-coated substrates with microtopography. Understanding such interactions could lead to new applications in e.g., medical implants as well as shed light on processes such as embryonic development, angiogenesis, wound healing, and tumor progression. In this work, the influence of surface topography and chemistry on $\alpha \mathrm{v} \beta 3$ and $\alpha 5 \beta 1$ integrinmediated cell adhesion and migration of healthy and malignant human cell types (human coronary artery endothelial cells, human osteosarcoma cells, and human skin fibroblasts cells) was studied, using microgrooved and flat substrates covered by two different extracellular proteins, fibronectin and vitronectin. Although some general behaviors can be observed, cell migration (speed, directionality, and persistence time) and morphological adaptation (cell area, aspect ratio, and circularity) of cells on protein-coated microgrooved substrates are mainly dependent on the cell type and its specific integrin expression.

\section{INTRODUCTION}

Integrins are cell membrane receptors that are activated by extracellular matrix (ECM) ligands. ${ }^{1-6}$ They serve as a mechanical anchorage and are significantly involved in the bidirectional interaction between the actin cytoskeleton and the ECM. ${ }^{7-10}$ The ligated receptors cluster and regulate chemical signaling by controlling the spatio-temporal assembly of adapters and enzymes. ${ }^{11}$ Studies on planar surfaces have demonstrated that such initial clustering results in focal complexes, which are small protein plaques, less than $1 \mu \mathrm{m}$ in size, that consist mainly of the integrin $\alpha \mathrm{v} \beta 3$, phosphotyrosine, and talin. ${ }^{12}$ The proper transmission of force through the actin cytoskeleton enables the maturation of focal complexes to focal adhesions (FAs). ${ }^{13-15}$ FAs are larger plaques than focal complexes, with sizes up to a few micrometers, that incorporate the integrin $\alpha 5 \beta 1$ and zyxin in addition to the components of focal complexes. ${ }^{12,16}$ In the last stage of maturation, fibrillar adhesions are formed, which are fibers that translocate centripetally across the ventral surface of the cell. Such fibers mainly contain $\alpha 5 \beta 1$ and tensin, ${ }^{16,17}$ and induce ECM remodeling, not only by changing the composition of the FAs but also of the cell environment. ${ }^{18,19}$ In contrast, the strengthening of the adhesion sites and the cellular mechanotransduction are attributed to $\alpha \mathrm{v} \beta 3$, which is confined to the edge of the cell membrane..$^{10,20,21}$ Integrins are thus involved in regulating the cell morphology, e.g., in protrusion formation, as well as in cell migration through the activation of the small GTPases Rac1 and RhoA, which induce lamellipodia formation and actin contraction, respectively. ${ }^{22}$ Moreover, the antagonization of $\alpha 5 \beta 1$-recycling loops and the overexpression of $\alpha \mathrm{v} \beta 3$ promote directionally persistent migration, ${ }^{23-25}$ which suggest that $\alpha 5 \beta 1$ induces random migration unlike $\alpha \mathrm{v} \beta 3 .{ }^{26}$ Nevertheless, recent studies have brought to light the complexity of such mechanisms, ${ }^{27,28}$ which

Received: December 22, 2018

Accepted: January 8, 2019

Published: January 22, 2019 
calls for new tools and strategies that could allow the study of integrins and their related cell substructures, i.e., lamellipodia, actin cytoskeleton, and FAs in well-defined environments. With this objective, substrates with controlled micro- and nanotopography consisting of different structures (grooves, pores, pillars, etc.) and materials (titanium, silicon, poly(dimethylsiloxane) (PDMS), etc.) have been designed by different techniques (photolithography, reactive ion etching, soft lithography, nanoimprint lithography, etc.). ${ }^{29}$ For example, PDMS substrates consisting of micro- and nanogrooves showed that fibroblast cells are sensitive to grooves with a minimal depth of $50 \mathrm{~nm}$, whereas endothelial cells require grooves about twice deeper to elicit a response, thus evidencing the importance of cell type to establish general observations. ${ }^{30}$ Moreover, different fabrication and functionalization techniques have been developed to achieve spatial and temporal control over the substrates used for cell culture. ${ }^{31-33}$ Thus, strategies to orthogonally functionalize substrates consisting of various materials have been developed based on their different chemistry achieving chemical control at the micro- and nanoscale, ${ }^{34-37}$ which e.g., demonstrated the tendency of the integrins $\alpha 5 \beta 1$ and $\alpha \mathrm{v} \beta 3$ to colocalize when using orthogonally functionalized micropatterns. ${ }^{34}$ Furthermore, the elucidation of these cell-material interactions may also result in medical advancements, e.g., in the treatment of cancer, due to the role of integrins in angiogenesis and tumor invasion. ${ }^{38-44}$ The development of implants is another medical field that would profit from a deeper understanding of integrinmediated cell migration, ${ }^{45-50}$ especially when using substrates with micro- and nanotopography. There is also a lack of knowledge regarding the influence of cell type on adhesion and migration, which should be remedied when considering medical applications, given that cells may possess and express different integrins, ${ }^{3}$ and thus, results obtained with a particular cell type may not be valid for the others.

In this work, we focused on studying the influence of combining specific topographies and coatings on $\alpha 5 \beta 1$ and $\alpha \mathrm{v} \beta 3$ integrin-mediated cell adhesion and migration across cells of different biological origins including healthy and malignant tissues as well as different functions in vivo to establish common traits. With this objective, the behavior of three different human cell lines, human bone osteosarcoma cells (U2OS), human skin fibroblast cells (FC), and human coronary artery endothelial cells (EC), was evaluated on microgrooved substrates consisting of parallel grooves with widths in the micron-scale and depths in the submicron-scale. Moreover, the substrates were coated with two ECM proteins, fibronectin (FN) and vitronectin (VN), which induce different integrin activation, i.e., FN activates both $\alpha \mathrm{v} \beta 3$ and $\alpha 5 \beta 1$ integrins, whereas VN activates $\alpha \mathrm{v} \beta 3$ but not $\alpha 5 \beta 1{ }^{4}$

\section{MATERIALS AND METHODS}

2.1. Substrate Preparation. Microgrooved substrates, consisting of arrays of parallel grooves, were fabricated by photolithography, where the lateral size of the grooves was determined. The height of the grooves was controlled by physical vapor deposition (PVD). Firstly, a layer of hexamethyldisilazane (Sigma-Aldrich) was applied to silicon wafers (Siegert Wafer $\mathrm{GmbH}$, Germany) after prebaking them for $30 \mathrm{~min}$ at $200{ }^{\circ} \mathrm{C}$, to improve the adhesion of the photoresist. Afterwards, the wafers were coated with the positive photoresist ma-P1210 (Micro Resist Technology $\mathrm{GmbH}$, Germany) to achieve a thickness of approximately
1.0-1.2 $\mu \mathrm{m}$, followed by a $1 \mathrm{~min}$ bake at $100{ }^{\circ} \mathrm{C}$. Coated wafers were aligned (MJB4, Süss MicroTech GmbH, Germany) to a chromium photomask (ML\&C GmbH, Germany) and exposed to UV light $(\lambda=405 \mathrm{~nm})$. After this process, the solubilized regions were removed with a developer solution (ma-D531, Micro Resist Technology $\mathrm{GmbH}$, Germany) and a chromium layer of the desired thickness was deposited via PVD. The remaining photoresist was then removed by sonication for $5 \mathrm{~min}$ in a remover solution $(\mathrm{mr}$ Rem 660, Micro Resist Technology GmbH, Germany). The silicon wafers with chromium microridges were used as templates for replica molding with Sylgard 184 (Dow Corning). PDMS was mixed at a 10:1 ratio (elastomer/curing agent) and incubated at $65^{\circ} \mathrm{C}$ for $24 \mathrm{~h}$ to obtain microgrooved PDMS substrates with $650 \mathrm{~nm}$ deep $(D)$ and $5 \mu$ m wide $(W)$ grooves. The PDMS substrates were characterized with a Zeiss Ultra 55 (Carl Zeiss, Germany) scanning electron microscope (Figure S1). Flat PDMS substrates were also produced and used as control.

2.2. Substrate Coating. Prior to cell seeding, the PDMS substrates were sterilized with an aqueous $70 \%$ ethanol solution. Afterwards, they were either incubated with $10 \mu \mathrm{g} /$ $\mathrm{mL}$ human fibronectin (FN) or with $2.25 \mu \mathrm{g} / \mathrm{mL}$ of bovine vitronectin (VN) (Sigma-Aldrich) for $30 \mathrm{~min}$ to achieve their successful physisorption on the PDMS. Washings were performed after each step with phosphate buffered saline (PBS).

2.3. Cell Culture. Human coronary artery endothelial cells (EC) were cultured in endothelial cell growth medium supplemented with supplement mix (Promocell, Germany) and $1 \%$ penicillin/streptomycin (Life Technologies). Human osteosarcoma cells (U2OS) and human skin fibroblast cells (FC) were cultured in Dulbecco's modified Eagle's medium GlutaMAX with $4.5 \mathrm{~g} / \mathrm{L}$ glucose and (-) pyruvate (Gibco) supplemented with $10 \%$ fetal bovine serum (Gibco) and $1 \%$ penicillin/streptomycin. EC below passage 7 were used to avoid dedifferentiation. The cells were maintained in an incubator at $37{ }^{\circ} \mathrm{C}, 5 \% \mathrm{CO}_{2}$, and high humidity (>90\%). Subculturing was performed with trypsin/ethylenediaminetetraacetic acid $0.04 \%$ (Promocell, Germany) before reaching $100 \%$ confluence.

2.4. Cell Seeding and Fixation. In all experiments, FC were seeded at a density of 15 cells $/ \mathrm{mm}^{2}, \mathrm{EC}$ at $20 \mathrm{cells} / \mathrm{mm}^{2}$, and $\mathrm{U} 2 \mathrm{OS}$ at 15 cells $/ \mathrm{mm}^{2}$ to avoid mutual disturbance during migration. After cell seeding for $72 \mathrm{~h}$, the cells were fixed on the substrates with paraformaldehyde (PFA 4\% in PBS, Serva Electrophoresis $\mathrm{GmbH}$, Germany). PFA was removed after exactly $15 \mathrm{~min}$ and the substrates were washed with PBS. The substrates were finally stored at $4{ }^{\circ} \mathrm{C}$ in the dark until staining.

2.5. Immunostaining. The cells were permeabilized by 3 min incubation in $0.1 \%$ Triton X-100 (Sigma-Aldrich) in PBS. They were then incubated for $30 \mathrm{~min}$ in $1 \%$ bovine serum albumin (BSA; Sigma-Aldrich). Afterwards, the cells that were stained for nuclei, actin filaments, and FAs were incubated for $1 \mathrm{~h}$ with mouse antipaxillin (BD Biosciences) at 1:400 dilution in PBS with $1 \%$ BSA. Then, they were incubated in a PBS solution of 4',6-diamidino-2-phenylindole dihydrochloride at a dilution of 1:1000 (Serva Electrophoresis GmbH, Germany), phalloidin tetramethylrhodamine (TRITC) at 1:50 (SigmaAldrich), and goat antimouse Alexa Fluor 488 (Life Technologies) diluted at 1:100 for $45 \mathrm{~min}$. Alternatively, the cells that were stained for specific integrins were incubated in a PBS solution with 1\% BSA containing 1:100 anti-integrin- 

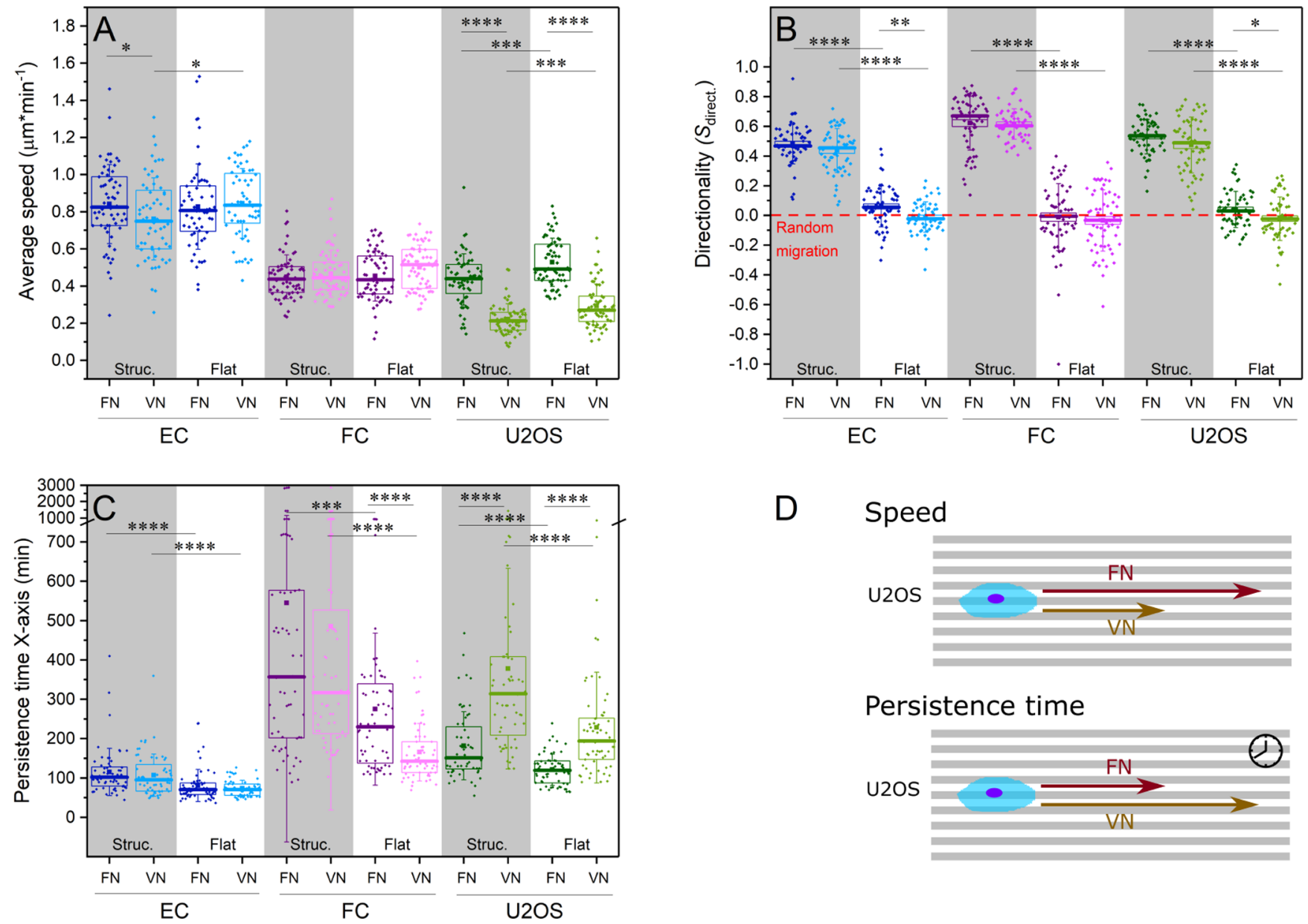

Speed

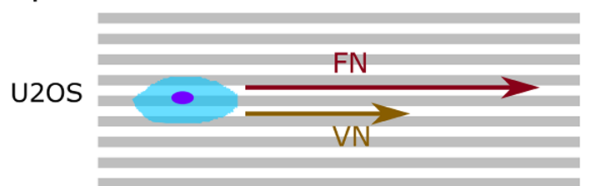

Persistence time

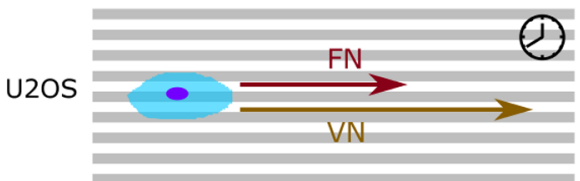

Figure 1. Cell migration parameters of the different cell types at different substrate topographical and coating conditions. (A) The average speed, (B) directionality, and (C) persistence time along the $X$-axis (microgroove direction) were measured for EC, FC, and U2OS on microgrooved ( $D=$ $650 \mathrm{~nm}, W=5 \mu \mathrm{m})$ and flat substrates coated with FN or VN $\left(N_{\text {cells }}=60 ; N_{\text {experiments }} \geq 3\right)$. (D) Schematic representation of coating-dependent speed and persistence time of cells migrating along the $X$-axis. Statistical significance was determined by the nonparametric Kruskal-Wallis ANOVA test $(* p<0.05, * * p<0.01, * * * p<0.001$, $* * * * p<0.0001)$.

$\alpha \mathrm{V} \beta 3$ (MAB1976, mouse antihuman, Millipore) and 1:100 anti-integrin- $\alpha 5$ antibodies (MAB11, rat antihuman, provided by Prof. Yamada, National Institute for Dental Research, Bethesda, MD) for $1 \mathrm{~h}$. The samples were subsequently incubated for $45 \mathrm{~min}$ in a solution of 1:50 goat antimouse Alexa Fluor 647 (Life Technologies) and 1:50 goat antirat Alexa Fluor 488 antibodies (Life Technologies) diluted in PBS with $1 \%$ BSA. All of the incubations with antibodies were performed at room temperature in wet chambers. Between incubations and prior to imaging, the samples were washed with PBS.

2.6. Image Acquisition. For cell migration studies, phase contrast images were acquired every $10 \mathrm{~min}$ for $24 \mathrm{~h}$ with an inverted microscope (Observer Z1, Carl Zeiss, Germany) and a $5 \times$ objective (N-Achroplan, Carl Zeiss, Germany). Fluorescence microscopy was used to visualize the actin cytoskeleton and paxillin-FAs in an inverted microscope Imager Z1 (Carl Zeiss, Germany) using 20x and $40 \times$ Achroplan objectives (Carl Zeiss, Germany). The specific integrin clusters were imaged with a $40 \times$ oil immersion objective (Leica HCX PL APO, Leica Camera AG, Germany) and a zoom factor of 2 in a confocal microscope (Leica TCS SP5 X, Leica Microsystems, Germany) equipped with a mercury metal halide bulb (Leica Camera AG, Germany).
2.7. Data Analysis and Statistics. All images were analyzed with Image ${ }^{51}$ and the resulting data were processed with Origin (OriginLab). Cell migration trajectories, from a total of 60 cells per experiment from three independent experiments, were manually tracked with an ImageJ plugin, MTrackJ, ${ }^{52}$ where the cell migration speed was calculated. Cell morphological parameters were calculated for at least 166 manually marked cells from at least three independent experiments for each experimental condition. The total area of $\alpha \mathrm{v} \beta 3$ and $\alpha 5 \beta 1$ FAs of 30 cells in total from 2 different experiments was automatically analyzed with ImageJ.

To calculate the average cell directionality, the following formula was used ${ }^{53}$

$$
S_{\text {direct. }}=\cos (2 \alpha)
$$

where $\alpha$ is the angle in radians between the first and last points of the track, and the direction of the grooves or the $X$-axis in flat substrates. $S_{\text {direct. }}=1$ is obtained when cells migrate parallel to the reference (groove direction), $S_{\text {direct. }}=0$ when they migrate randomly, and $S_{\text {direct. }}=-1$ when cells migrate perpendicular to the reference.

Cell aspect ratio (AR) was calculated as follows 


$$
\mathrm{AR}=\frac{A_{\text {maj }}}{A_{\text {min }}}
$$

$A_{\text {maj }}$ and $A_{\text {min }}$ represents the major and minor axes, respectively, of a fitted ellipse on the cell.

Migration persistence time was calculated by dividing the total time that cells migrated $(24 \mathrm{~h})$ by the number of times cells changed the direction of migration in each axis, as previously described (Figure S2). ${ }^{54}$

Boxplots were used to represent the distribution of values obtained for the cell morphometric and migration parameters as well as the integrin-FA area. The average value $(\square)$, the median (horizontal line in the middle of the box), the interquartile range (IQR, "middle fifty", illustrated as the box), and one positive and negative standard deviation presented by the whiskers of the IQR of each data set are provided.

To corroborate the results, statistical significance was determined by the nonparametric Kruskal-Wallis analysis of variance (ANOVA) test (ns: not significant, $* p<0.05$, ** $p<$ $0.01, * * * p<0.001, * * * * p<0.0001)$.

\section{RESULTS AND DISCUSSION}

3.1. Effect of Substrate Topography and Coating on Cell Migration. Cell migration speed, directionality, and persistence of the three cell types (U2OS, FC, EC) on two different ECM protein coatings (VN and FN) of flat and microgrooved substrates $(D=650 \mathrm{~nm}, W=5 \mu \mathrm{m})$ were analyzed (Figures 1 and S3). The depth of the microgrooves was inspired on the sizes of collagen bundles in vivo ${ }^{55}$ and specifically chosen to ensure a high response in terms of morphology adaptation and migratory behavior of the cells. ${ }^{56-58}$ Moreover, a width of $5 \mu \mathrm{m}$ was selected to avoid the confinement growth of FAs, ${ }^{59}$ which usually present a smaller size. ${ }^{60,61}$

As shown in Figure 1A, the migration speeds of EC (0.75$0.84 \mu \mathrm{m} / \mathrm{min}$ ) are higher than the ones exhibited by FC $(0.43-0.52 \mu \mathrm{m} / \mathrm{min})$ and U2OS $(0.21-0.49 \mu \mathrm{m} / \mathrm{min})$. EC migrated significantly slower on microgrooved substrates than on the flat ones when coated with $\mathrm{VN}$. In contrast, no differences were observed on FN coatings. On structured substrates, EC migrated faster on FN-coated than on $\mathrm{VN}$ coated surfaces, whereas EC on flat substrates showed no differences. FC did not exhibit any significant change in migration speed due to topography or protein coating, in contrast to U2OS, which showed remarkable coating- and topography-dependent differences. On both coatings, U2OS migrated significantly slower on microgrooves than on flat substrates. Additionally, the speed of this cell type was severely reduced (by $50 \%$ or more) on $\mathrm{VN}$ in comparison with $\mathrm{FN}$ coatings, both on microgrooved and flat substrates (Figure 1A). Thus, cellular responses are highly dependent on the cell type. In responsive cells, microgrooves and $\mathrm{VN}$-coating tend to reduce the migration speed.

Additionally, the cells were observed to migrate preferentially along the direction of the microgrooves on structured substrates, as previously reported. ${ }^{62,63}$ More specifically, a median directionality value $\left(S_{\text {direct. }}\right)$ higher than 0.45 was obtained under all the conditions, whereas on flat substrates, the cells migrated randomly $\left(S_{\text {direct. }} \approx 0\right)$, in the absence of a guiding signal (Figure $1 \mathrm{~B})$. The maximum median $S_{\text {direct. }}$ is observed with FC, with $S_{\text {direct. }} \approx 0.63$, whereas for the other two cell types, $S_{\text {direct. }}$ is below 0.55 . These results are in agreement with the reported observation that FC migrated
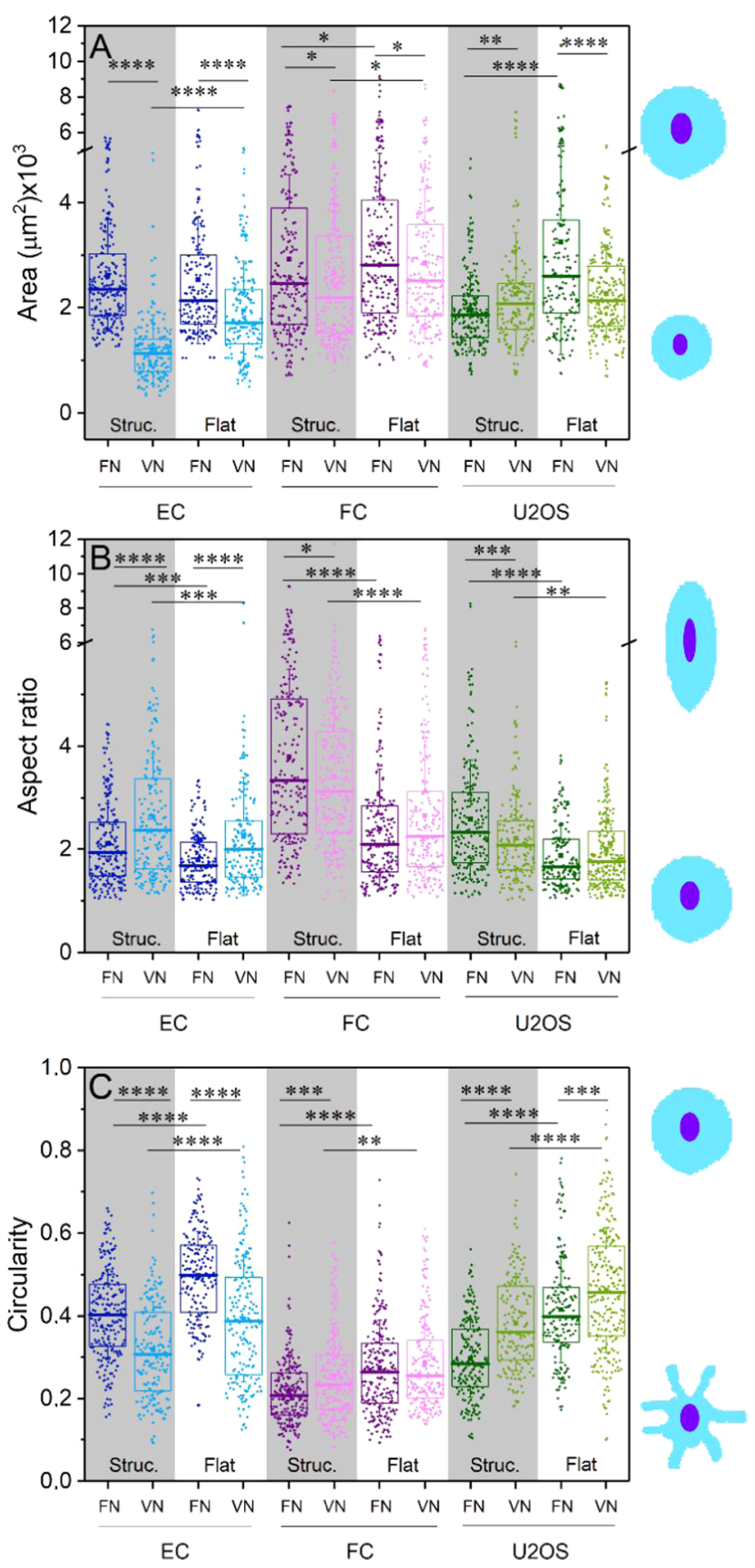

Figure 2. Analysis of cell morphometric parameters. (A) Area, (B) aspect ratio, and $(C)$ circularity were analyzed for all three cell types (EC, FC, U2OS) on microgrooved and flat substrates coated with FN or VN $\left(N_{\text {cells }} \geq 166 ; N_{\text {experiments }} \geq 3\right)$. Statistical significance was determined by the nonparametric Kruskal-Wallis ANOVA test $\left({ }^{*} p<\right.$ $0.05, * * p<0.01, * * * p<0.001, * * * * p<0.0001)$.

more directionally along the microgrooves of $D=200 \mathrm{~nm}$ and $W=2 \mu \mathrm{m}$ than EC. ${ }^{30}$ No significant differences were observed on structured substrates between coatings regardless of cell type, indicating no influence on cell contact guidance due to surface coating. In contrast, EC and U2OS seeded on flat substrates showed slightly higher directionality on FN- than on VN-coated substrates. Migration directionality is therefore highly influenced by the presence of microgrooves. In their absence, the results vary depending on the cell type and surface coating. 

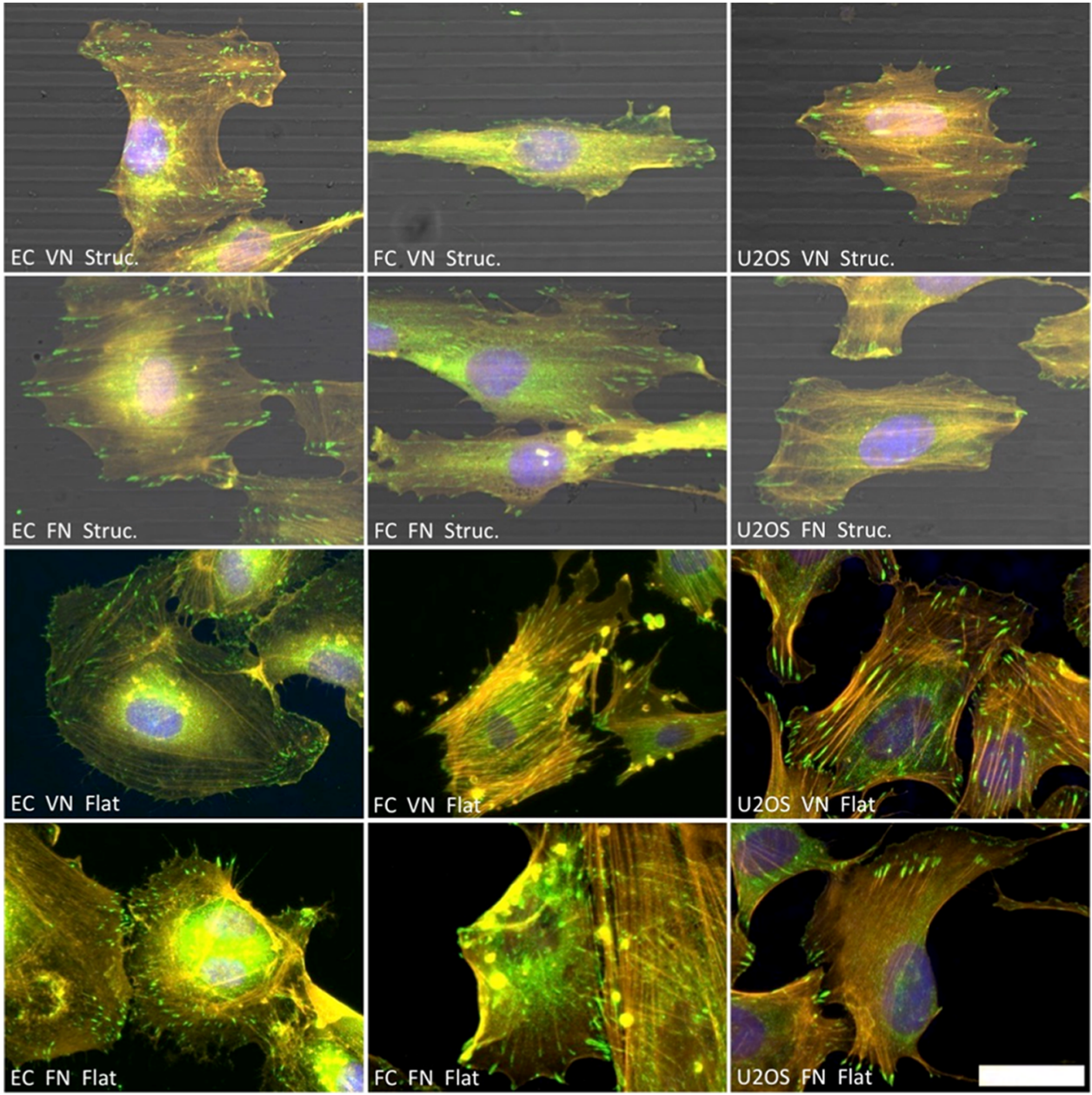

Figure 3. Analysis of EC, FC, and U2OS cell internal structures by fluorescence microscopy. Actin cytoskeleton (yellow), nuclei (blue), and FAs (green) of cells fixed at $48 \mathrm{~h}$ after seeding on microgrooved and flat substrates coated with FN or VN. Cells were immunostained with Alexa Fluor TRITC phalloidin (actin cytoskeleton), Hoechst (nuclei), and antipaxillin combined with an Alexa Fluor 488 secondary antibody (FAs). Scale bar $=35 \mu \mathrm{m}$.

To determine if surface coating influences migration persistence, which is related to the cell steering mechanisms, ${ }^{64}$ the cell persistence time was analyzed (Figure 1C). All cell types migrated significantly longer time along the $X$-axis on microgrooved substrates, i.e., along the microgrooves, than on flat surfaces independent of the protein coating. Thus, microgrooves favor longer migration times along them. Additionally, U2OS migrated for longer times on $\mathrm{VN}$-coated surfaces than on the FN-coated ones when the same topographical condition was used, whereas the opposite trend was observed for FC on flat substrates. Along the $Y$ axis (perpendicular to the microgrooves), no clear tendency was observed regarding the differences in persistence time between microgrooved and flat substrates for a given coating (Figure S3). These results indicate that microgrooves guide the cell steering system, increasing the cell migration persistence, mainly along their axis. Moreover, the effect of surface coating varies across cell types, U2OS being the most sensitive cell type to this parameter.

By comparing both coatings ( $\mathrm{FN}$ and $\mathrm{VN}$ ) in each surface topography condition (microgrooved and flat substrates), we observed that cell migration is highly dependent on cell type, which could explain the differences reported in the literature ${ }^{23,25-28}$ and could be caused by the different set of integrins expressed by each cell type. ${ }^{3}$ Nevertheless, some general trends were observed, such as the clear influence of microgrooves on directionality and persistence, with the cells mainly migrating along the axis of the grooves. Moreover, the previously observed correlation between cell migration speed and persistence time was confirmed for U2OS. ${ }^{54}$ The faster the cells migrate, the smaller their persistence time is (Figure 1D).

\subsection{Effect of Substrate Topography and Coating on} Cell Morphometric Parameters Related to Cell Adhesion. To further investigate the role of surface coating on cells, the morphological adaptation of EC, FC, and U2OS on the different substrates was studied (Figure 2).

FC showed the largest median areas (2193-2812 $\left.\mu \mathrm{m}^{2}\right)$ followed by the U2OS $\left(1867-2610 \mu \mathrm{m}^{2}\right)$ and the EC (1139$2354 \mu \mathrm{m}^{2}$ ) due to their different biological nature (Figure $2 \mathrm{~A}$ ). All cell lines showed an equal or larger area on flat substrates than on microgrooved ones. Moreover, this area was smaller on VN-coated than on FN-coated substrates. The only exception to this observation was the U2OS seeded on microgrooved substrates, where the $\mathrm{VN}$ coating promotes significantly higher cell areas than the FN coating. Thus, all cell lines adapted their area size to the chemistry and topography of the surface. 

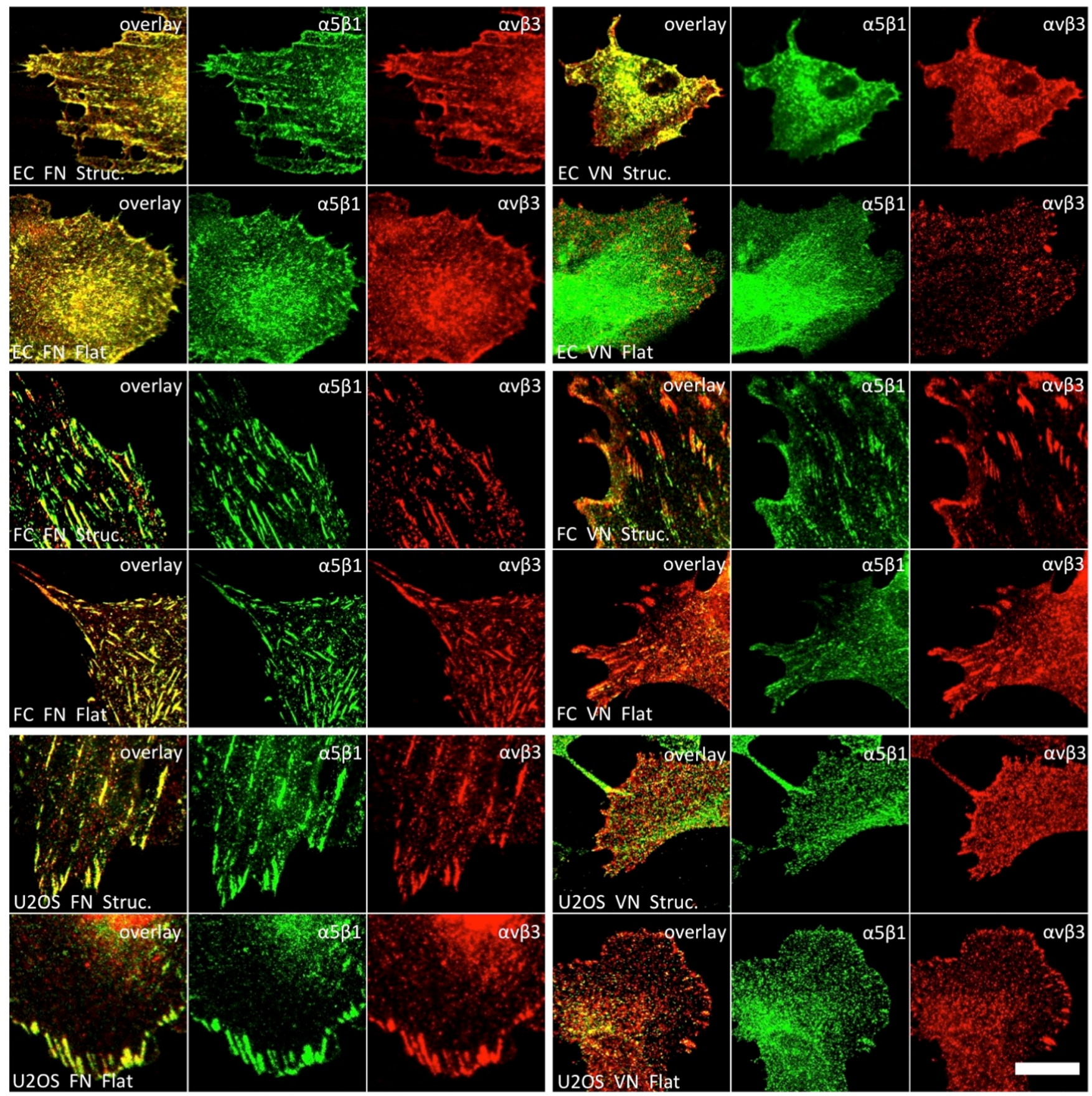

Figure 4. Analysis of FA composition in EC, FC, and U2OS by fluorescence microscopy. Cells were fixed and immunostained for $\alpha 5$ (green) and $\alpha \mathrm{v} \beta 3$ (red) integrins at $48 \mathrm{~h}$ after seeding on flat and microgrooved substrates coated with FN or VN. Scale bar $=20 \mu \mathrm{m}$.

Additionally, cell elongation was analyzed through the cell aspect ratio (AR). A significant increase of the median AR of cells on microgrooved substrates (3.34-1.93) in comparison with flat surfaces $(2.25-1.66)$ was observed (Figure 2B). On microgrooved substrates, both FC and U2OS were significantly more elongated on $\mathrm{FN}$-coated substrates than on $\mathrm{VN}$-coated ones, whereas no significant differences in cell elongation due to substrate coating were observed in these two cell types on flat surfaces. EC instead were significantly more elongated on $\mathrm{VN}$-coated microgrooved and flat substrates than on $\mathrm{FN}$ coated ones. As previously observed, FC elongated more on microgrooves than EC. ${ }^{30}$
By analyzing the cell circularity, which is proportional to the area divided by the square of the perimeter, we observed in both coating conditions that all cell types show a significantly higher median circularity on flat $(0.50-0.26)$ than on microgrooved substrates $(0.41-0.22)$ (Figure 2C). FC and U2OS were significantly more circular on VN-coated substrates than on the FN-coated ones, except for FC on flat substrates, where no coating-dependent differences in circularity were observed. Conversely, EC median circularity was significantly higher on FN-coated substrates than on the VNcoated ones.

As observed in cell migration, cell morphology is clearly affected by the presence of microgrooves. Specifically, cells 

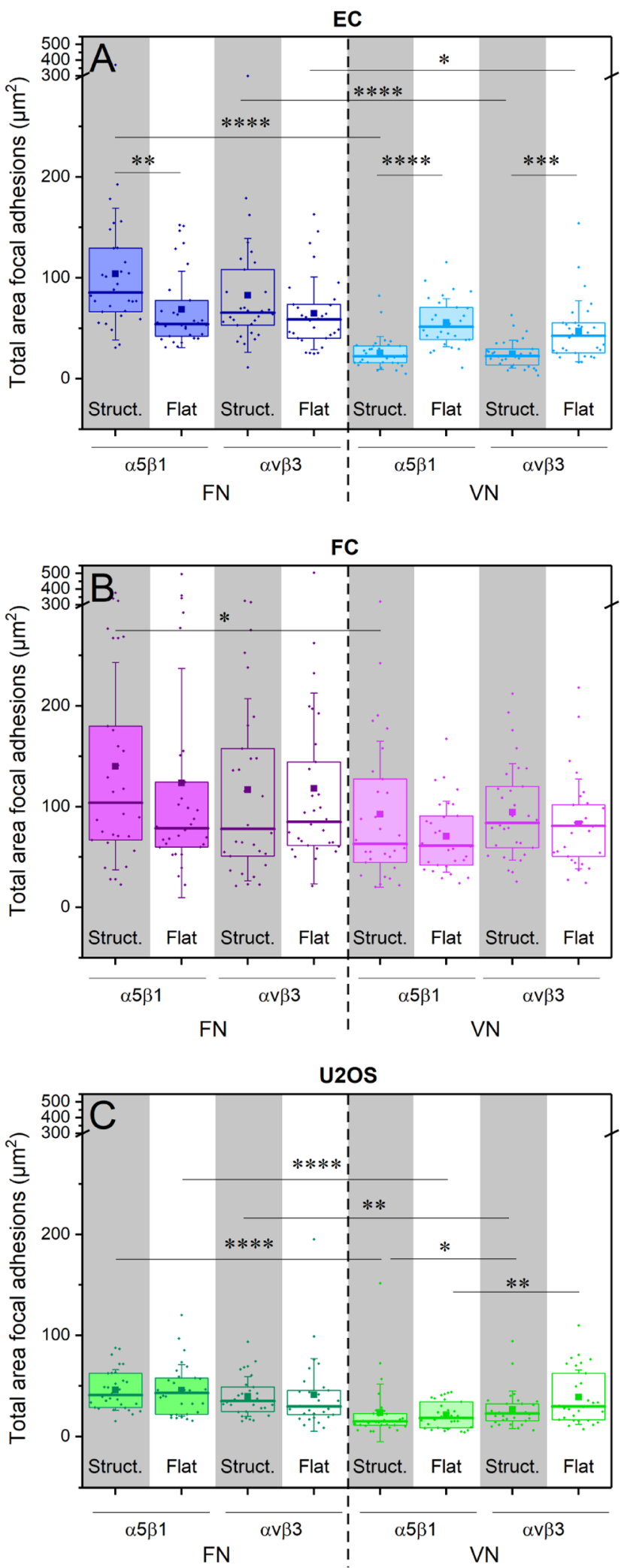

Figure 5. Analysis of $\alpha \mathrm{v} \beta 3$ and $\alpha 5 \beta 1$ FA areas. Quantification of $\alpha \mathrm{v} \beta 3$ and $\alpha 5 \beta 1 \mathrm{FA}$ areas of (A) EC, (B) FC, and (C) U2OS on flat and microgrooved substrates coated with $\mathrm{VN}$ and $\mathrm{FN}\left(N_{\mathrm{FAs}} \geq 30\right.$; $N_{\text {experiments }} \geq 2$ ). Statistical significance was determined by the nonparametric Kruskal-Wallis ANOVA test $(* p<0.05$, $* * p<0.01$, $* * * p<0.001, * * * * p<0.0001)$. seeded on flat substrates resulted in larger cell areas with lower elongation and higher circularity than cells seeded on microgrooved substrates. Additionally, the response to surface coating was highly dependent on cell type, probably due to the different integrin expression patterns of cells.

3.3. Integrin Expression in FAs of Cells on ProteinCoated Microgrooved Substrates. As mentioned above, cell adhesion and migration are known to be mediated by internal structures related to mechanotransduction such as the actin cytoskeleton and FAs. However, the influence of topography on these structures remains controversial. For example, some reports showed an increase of FA size on flat surfaces in comparison with microgrooved or nanostructured substrates, ${ }^{65,66}$ whereas some others showed the opposite. $^{57,67,68}$ To shed light on this, the actin cytoskeleton and FAs were qualitatively analyzed.

As shown in Figure 3, an increased number and size of FAs and actin stress fibers (SFs) were observed for all cell types on flat substrates in comparison with microgrooved substrates for any given ECM protein coating. Thus, the influence of topography on cell behavior could also be confirmed at the subcellular level.

To explain the differences related to surface coating and topography, we analyzed the presence of specific integrins, namely $\alpha \mathrm{v} \beta 3$ and $\alpha 5 \beta 1$, in the FAs (Figures 4 and 5). EC exhibited significantly larger $\alpha 5 \beta 1$ and $\alpha \mathrm{v} \beta 3$ FAs on FN-coated than on VN-coated microgrooved substrates. Moreover, on FN-coated substrates, the $\alpha 5 \beta 1 \mathrm{FA}$ area on microgrooved substrates was significantly larger than that of flat substrates. On VN-coated substrates, $\alpha v \beta 3$ FAs were significantly larger on flat than on structured substrates. The FA area of FC did not significantly change due to chemical composition or substrate topography, with the only exception of $\alpha 5 \beta 1$ FAs which were significantly larger on FN-coated microgrooved substrates than on the $\mathrm{VN}$-coated ones, as expected given that VN triggers $\alpha \mathrm{v} \beta 3$. $^{4,34,49} \mathrm{U} 2 \mathrm{OS}$ showed significantly larger $\alpha \mathrm{v} \beta 3$ FAs than $\alpha 5 \beta 1$ on both VN-coated flat and microgrooved substrates. Instead, on FN-coated microgrooved and flat substrates, no differences in the FA area was observed between integrins. Moreover, FAs of both integrin types were significantly larger on FN-coated substrates than on the VNcoated ones, except for $\alpha \mathrm{v} \beta 3$ FAs on flat substrates where no significant differences were found. Surprisingly though, the VN-coated substrates, especially for EC, appeared to show similar sizes for $\alpha 5 \beta 1$ than for $\alpha \mathrm{v} \beta 3$ FAs. However, although $\alpha \mathrm{v} \beta 3$ expression mainly comes from clusters located at the cell periphery as expected, the $\alpha 5 \beta 1$ expression mainly consists of speckles at the perinuclear area (Figure 4). Thus, the apparently high $\alpha 5 \beta 1$ results can be explained by software overevaluation of the central cell areas.

In summary, we can state that all three cell types exhibited particularities in the adaptation of the FA composition to the different substrate topographies, as mainly observed in the cell migration and morphological studies. However, the migration and morphological parameters that were clearly impacted by topography such as migration directionality or cell circularity could not be clearly correlated with integrin expression, although previous reports indicated that " $2.5 \mathrm{D}$ " and $3 \mathrm{D}$ structures modify FA and integrin distribution. ${ }^{69,70}$ Conversely, other parameters were mainly affected by protein coating such as migration speed, which could be therefore mostly related to $\alpha 5 \beta 1$ and $\alpha \mathrm{v} \beta 3$ expression. For example, the higher speed observed for $\mathrm{U} 2 \mathrm{OS}$ on $\mathrm{FN}$ - than $\mathrm{VN}$-coated flat and 
microgrooved substrates correlated with the larger FA total area, as previously observed. ${ }^{24,61,71,72}$ Moreover, this area can be associated with the expression of $\alpha 5 \beta 1$, given that $\alpha \mathrm{v} \beta 3$ is also expressed on VN-coated substrates. ${ }^{4,34,49}$ Thus, migration speed is probably boosted by $\alpha 5 \beta 1$ and its role activating RhoA, which results in the development of actin SFs. ${ }^{73,74}$ However, the higher cell persistence observed by U2OS on $\mathrm{VN}$-coated substrates is not supported by a clear $\alpha \mathrm{v} \beta 3 \mathrm{FA}$ area enhancement. Thus, we believe that other structures, for example, micropatterns with deeper grooves could be explored in the future to further study the interaction between surface topography and surface chemistry on integrin-mediated cell adhesion and migration of different cell types. Possibly, the common traits found across the experiments could be explained by the shared biological functions associated with e.g., the adherent character of the studied cell types, whereas noncommon biological functions may result in different mechanisms. ${ }^{75,76}$ Indeed, different biological functions associated with either healthy or malignant tissues are determined by different integrin expressions, ${ }^{79}$ e.g., white blood cells even possess specific integrins that are not expressed in other mammalian cells. ${ }^{3}$ Additionally, the influence of other parameters such as surface stiffness might also play a role, ${ }^{77-80}$ e.g., its relevance was shown in force generation, actin flow, and integrin expression of certain subtypes such as $\alpha 5 \beta 1$ and $\alpha \mathrm{v} \beta 6$. $^{77}$ Thus, the effect of surface stiffness on $\alpha 5 \beta 1$ and $\alpha \mathrm{v} \beta 3$-integrin mediated cell adhesion and migration might be systematically studied in the future.

\section{CONCLUSIONS}

The role of surface coating (FN or VN) and topography (flat or microgrooved polymeric substrates) in integrin-mediated cell adhesion and migration of different human cell types (EC, FC, U2OS) has been analyzed. It can be concluded that although some general behaviors are acknowledged, cell migration (speed, directionality, and persistence time) and morphological adaptation (cell area, and especially aspect ratio and circularity) are highly dependent on the cell type and its particular integrin expression. Nevertheless, further studies varying the sizes of the micropatterns, e.g., increasing the depth of the grooves, could result in clearer interactions between surface topography and chemistry. Thus, the protein-coated micropatterns are a promising tool to continue shedding light on the intricate roles of $\alpha \mathrm{v} \beta 3$ and $\alpha 5 \beta 1$ integrins, which might eventually give some new insights into the integrin-related biomedical industry.

\section{ASSOCIATED CONTENT}

\section{S Supporting Information}

The Supporting Information is available free of charge on the ACS Publications website at DOI: 10.1021/acsomega.8b03608.

SEM image of a PDMS microgrooved substrate; representation of cell migration changes in $X$ - and $Y$ axes; migration persistence along the $Y$-axis (PDF)

\section{AUTHOR INFORMATION}

\section{Corresponding Authors}

*E-mail: adria.sales@grenoble-inp.fr (A.S.).

*E-mail: jguasch@icmab.es (J.G.).

ORCID

Jaume Veciana: 0000-0003-1023-9923
Imma Ratera: 0000-0002-1464-9789

Judith Guasch: 0000-0002-3571-4711

\section{Present Addresses}

\#Univ. Grenoble Alpes, CNRS, Grenoble INP, LMGP, F38000 Grenoble, France (A.S.).

${ }^{\nabla}$ Reutlingen University, Alteburgstrasse 150, D-72762 Reutlingen, Germany (K.E.).

\section{Author Contributions}

The manuscript was written through contributions of all authors. All authors have given approval to the final version of the manuscript.

\section{Notes}

The authors declare no competing financial interest.

\section{ACKNOWLEDGMENTS}

We kindly acknowledge D. P. Rosenblatt for proofreading the manuscript. This research was supported by the People Programme (Marie Curie Actions) of the 7th Framework Programme of the European Union (FP7/2007-2013) under the grant agreement Nr. 600388 of REA and the Agency for Business Competitiveness ACCIÓ through a Tecniospring fellowship (TECSPR15-1-0015). This work was also funded by the Max Planck Society through the Max Planck Partner Group "Dynamic Biomimetics for Cancer Immunotherapy" in collaboration with the Max Planck for Medical Research. The Spanish Ministry of Economy and Competitiveness (MAT2016-80826-R), the Networking Research Center on Bioengineering, Biomaterials, and Nanomedicine (CIBERBBN), and GenCat (grant 2017-SGR 918 and CERCA Programme) also supported this research. ICMAB acknowledges the support from the Spanish Ministry of Economy and Competitiveness, through the "Severo Ochoa" Programme for Centres of Excellence in R\&D (SEV-2015-0496).

\section{REFERENCES}

(1) Hynes, R. O. Integrins: Versatility, Modulation, and Signaling in Cell Adhesion. Cell 1992, 69, 11-25.

(2) Giancotti, F. G.; Ruoslahti, E. Integrin Signaling. Science 1999, $285,1028-1032$.

(3) Hynes, R. O. Integrins: Bidirectional, Allosteric Signaling Machines. Cell 2002, 110, 673-687.

(4) Humphries, J. D.; Byron, A.; Humphries, M. J. Integrin Ligands at a Glance. J. Cell Sci. 2006, 119, 3901-3903.

(5) Harburger, D. S.; Calderwood, D. A. Integrin Signalling at a Glance. J. Cell Sci. 2009, 122, 159-163.

(6) Barczyk, M.; Carracedo, S.; Gullberg, D. Integrins. Cell Tissue Res. 2010, 339, 269-280.

(7) Kuo, J.-C. Mechanotransduction at Focal Adhesions: Integrating Cytoskeletal Mechanics in Migrating Cells. J. Cell. Mol. Med. 2013, 17, 704-712.

(8) Geiger, B.; Spatz, J. P.; Bershadsky, A. D. Environmental Sensing through Focal Adhesions. Nat. Rev. Mol. Cell Biol. 2009, 10, 21-33.

(9) Roca-Cusachs, P.; Rio, A. D.; Puklin-Faucher, E.; Gauthier, N. C.; Biais, N.; Sheetz, M. P. Integrin-Dependent Force Transmission to the Extracellular Matrix by $\alpha$-Actinin Triggers Adhesion Maturation. Proc. Natl. Acad. Sci. U.S.A 2013, E1361-E1370.

(10) Roca-Cusachs, P.; Gauthier, N. C.; Rio, A. D.; Sheetz, M. P. Clustering of Alpha(5)Beta(1) Integrins Determines Adhesion Strength Whereas Alpha(V)Beta(3) and Talin Enable Mechanotransduction. Proc. Natl. Acad. Sci. U.S.A 2009, 106, 16245-16250.

(11) Morgan, M. R.; Byron, A.; Humphries, M. J.; Bass, M. D. Giving Off Mixed Signals-Distinct Functions of $\alpha 5 \beta 1$ and $\alpha \mathrm{v} \beta 3$ Integrins in Regulating Cell Behaviour. IUBMB Life 2009, 61, 731738 . 
(12) Zaidel-Bar, R.; Ballestrem, C.; Kam, Z.; Geiger, B. Early Molecular Events in the Assembly of Matrix Adhesions at the Leading Edge of Migrating Cells. J. Cell Sci. 2003, 116, 4605-4613.

(13) Galbraith, C. G.; Yamada, K. M.; Sheetz, M. P. The Relationship between Force and Focal Complex Development. J. Cell Biol. 2002, 159, 695-705.

(14) Katsumi, A.; Orr, A. W.; Tzima, E.; Schwartz, M. A. Integrins in Mechanotransduction. J. Biol. Chem. 2004, 279, 12001-12004.

(15) Katsumi, A.; Naoe, T.; Matsushita, T.; Kaibuchi, K.; Schwartz, M. A. Integrin Activation and Matrix Binding Mediate Cellular Responses to Mechanical Stretch. J. Biol. Chem. 2005, 280, 1654616549 .

(16) Zamir, E.; Katz, M.; Posen, Y.; Erez, N.; Yamada, K. M.; Katz, B.-Z.; Lin, S.; Lin, D. C.; Bershadsky, A.; Kam, Z.; Geiger, B. Dynamics and Segregation of Cell-Matrix Adhesions in Cultured Fibroblasts. Nat. Cell Biol. 2000, 2, 191-196.

(17) Pankov, R.; Cukierman, E.; Katz, B.-Z.; Matsumoto, K.; Lin, D. C.; Lin, S.; Hahn, C.; Yamada, K. M. Integrin Dynamics and Matrix Assembly: Tensin-Dependent Translocation of $\alpha 5 \beta 1$ Integrins Promotes Early Fibronectin Fibrillogenesis. J. Cell Biol. 2000, 148, $1075-1090$

(18) Katz, B.-Z.; Zamir, E.; Bershadsky, A.; Kam, Z.; Yamada, K. M.; Geiger, B. Physical State of the Extracellular Matrix Regulates the Structure and Molecular Composition of Cell-Matrix Adhesions. Mol. Biol. Cell 2000, 11, 1047-1060.

(19) Danen, E. H. J.; Sonneveld, P.; Brakebusch, C.; Fässler, R.; Sonnenberg, A. The Fibronectin-Binding Integrins $\alpha 5 \beta 1$ and $\alpha \mathrm{v} \beta 3$ Differentially Modulate RhoA-GTP Loading, Organization of Cell Matrix Adhesions, and Fibronectin Fibrillogenesis. J. Cell Biol. 2002, 159, 1071-1086.

(20) Rossier, O.; Octeau, V.; Sibarita, J.-B.; Leduc, C.; Tessier, B.; Nair, D.; Gatterdam, V.; Destaing, O.; Albigès-Rizo, C.; Tampé, R.; Cognet, L.; Choquet, D.; Lounis, B.; Giannone, G. Integrins $\beta 1$ and $\beta 3$ Exhibit Distinct Dynamic Nanoscale Organizations inside Focal Adhesions. Nat. Cell Biol. 2012, 14, 1057-1067.

(21) Schiller, H. B.; Hermann, M.-R.; Polleux, J.; Vignaud, T.; Zanivan, S.; Friedel, C. C.; Sun, Z.; Raducanu, A.; Gottschalk, K.-E.; Théry, M.; Mann, M.; Fässler, R. $\beta 1$ - and $\alpha \mathrm{v}$-Class Integrins Cooperate to Regulate Myosin II During Rigidity Sensing of Fibronectin-Based Microenvironments. Nat. Cell Biol. 2013, 15, 625-636.

(22) Burridge, K.; Wennerberg, K. Rho and Rac Take Center Stage. Cell 2004, 116, 167-179.

(23) Danen, E. H. J.; van Rheenen, J.; Franken, W.; Huveneers, S.; Sonneveld, P.; Jalink, K.; Sonnenberg, A. Integrins Control Motile Strategy through a Rho-Cofilin Pathway. J. Cell Biol. 2005, 169, $515-526$.

(24) Worth, D. C.; Hodivala-Dilke, K.; Robinson, S. D.; King, S. J.; Morton, P. E.; Gertler, F. B.; Humphries, M. J.; Parsons, M. $\alpha \mathrm{v} \beta 3$ Integrin Spatially Regulates VASP and RIAM to Control Adhesion Dynamics and Migration. J. Cell Biol. 2010, 189, 369-383.

(25) White, D. P.; Caswell, P. T.; Norman, J. C. $\alpha \mathrm{v} \beta 3$ and $\alpha 5 \beta 1$ Integrin Recycling Pathways Dictate Downstream Rho Kinase Signaling to Regulate Persistent Cell Migration. J. Cell Biol. 2007, $177,515-525$.

(26) Bridgewater, R. E.; Norman, J. C.; Caswell, P. T. Integrin Trafficking at a Glance. J. Cell Sci. 2012, 125, 3695-3701.

(27) Missirlis, D.; Haraszti, T.; Scheele, C. v. C.; Wiegand, T.; Diaz, C.; Neubauer, S.; Rechenmacher, F.; Kessler, H.; Spatz, J. P. Substrate Engagement of Integrins $\alpha 5 \beta 1$ and $\alpha \mathrm{v} \beta 3$ Is Necessary, but Not Sufficient, for High Directional Persistence in Migration on Fibronectin. Sci. Rep. 2016, 6, No. 23258.

(28) Shafaq-Zadah, M.; Gomes-Santos, C. S.; Bardin, S.; Maiuri, P.; Maurin, M.; Iranzo, J.; Gautreau, A.; Lamaze, C.; Caswell, P.; Goud, B.; Johannes, L. Persistent Cell Migration and Adhesion Rely on Retrograde Transport of Beta1 Integrin. Nat. Cell Biol. 2016, 18, 5464.

(29) Greiner, A. M.; Sales, A.; Chen, H.; Biela, S. A.; Kaufmann, D.; Kemkemer, R. Nano- and Microstructured Materials for in Vitro
Studies of the Physiology of Vascular Cells. Beilstein J. Nanotechnol. 2016, 7, 1620-1641.

(30) Biela, S. A.; Su, Y.; Spatz, J. P.; Kemkemer, R. Different Sensitivity of Human Endothelial Cells, Smooth Muscle Cells and Fibroblasts to Topography in the Nano-Micro Range. Acta Biomater. 2009, 5, 2460-2466.

(31) Kyvik, A. R.; Luque-Corredera, C.; Pulido, D.; Royo, M.; Veciana, J.; Guasch, J.; Ratera, I. Stimuli-Responsive Functionalization Strategies to Spatially and Temporally Control Surface Properties: Michael Vs. Diels Alder Type Additions. J. Phys. Chem. B 2018, 122, 4481-4490.

(32) Tatkiewicz, W. I.; Seras-Franzoso, J.; Garcia-Fruitós, E.; Vazquez, E.; Kyvik, A. R.; Guasch, J.; Villaverde, A.; Veciana, J.; Ratera, I. Surface-Bound Gradient Deposition of Protein Nanoparticles for Cell Motility Studies. ACS Appl. Mater. Interfaces 2018, 10, 25779-25786.

(33) Tatkiewicz, W. I.; Seras-Franzoso, J.; García-Fruitós, E.; Vazquez, E.; Ventosa, N.; Peebo, K.; Ratera, I.; Villaverde, A.; Veciana, J. Two-Dimensional Microscale Engineering of ProteinBased Nanoparticles for Cell Guidance. ACS Nano 2013, 7, 47744784.

(34) Guasch, J.; Conings, B.; Neubauer, S.; Rechenmacher, F.; Ende, K.; Rolli, C. G.; Kappel, C.; Schaufler, V.; Micoulet, A.; Kessler, H.; Boyen, H.-G.; Cavalcanti-Adam, E. A.; Spatz, J. P. Segregation Versus Colocalization: Orthogonally Functionalized Binary Micropatterned Substrates Regulate the Molecular Distribution in Focal Adhesions. Adv. Mater. 2015, 27, 3737-3747.

(35) Schenk, F. C.; Boehm, H.; Spatz, J. P.; Wegner, S. V. DualFunctionalized Nanostructured Biointerfaces by Click Chemistry. Langmuir 2014, 30, 6897-6905.

(36) Guasch, J.; Muth, C. A.; Diemer, J.; Riahinezhad, H.; Spatz, J. P. Integrin-Assisted T-Cell Activation on Nanostructured Hydrogels. Nano Lett. 2017, 17, 6110-6116.

(37) Guasch, J.; Diemer, J.; Riahinezhad, H.; Neubauer, S.; Kessler, H.; Spatz, J. P. Synthesis of Binary Nanopatterns on Hydrogels for Initiating Cellular Responses. Chem. Mater. 2016, 28, 1806-1815.

(38) Sheppard, D. Endothelial Integrins and Angiogenesis: Not So Simple Anymore. J. Clin. Invest. 2002, 110, 913-914.

(39) Ng, T.; Shima, D.; Squire, A.; Bastiaens, P. I. H.; Gschmeissner, S.; Humphries, M. J.; Parker, P. J. PKC $\alpha$ Regulates $\beta 1$ IntegrinDependent Cell Motility through Association and Control of Integrin Traffic. EMBO J. 1999, 18, 3909-3923.

(40) Parsons, M.; Keppler, M. D.; Kline, A.; Messent, A.; Humphries, M. J.; Gilchrist, R.; Hart, I. R.; Quittau-Prevostel, C.; Hughes, W. E.; Parker, P. J.; Ng, T. Site-Directed Perturbation of Protein Kinase C-Integrin Interaction Blocks Carcinoma Cell Chemotaxis. Mol. Cell. Biol. 2002, 22, 5897-5911.

(41) Caswell, P. T.; Spence, H. J.; Parsons, M.; White, D. P.; Clark, K.; Cheng, K. W.; Mills, G. B.; Humphries, M. J.; Messent, A. J.; Anderson, K. I.; McCaffrey, M. W.; Ozanne, B. W.; Norman, J. C. Rab25 Associates with $\alpha 5 \beta 1$ Integrin to Promote Invasive Migration in 3D Microenvironments. Dev. Cell 2007, 13, 496-510.

(42) Pallarola, D.; Platzman, I.; Bochen, A.; Cavalcanti-Adam, E. A.; Axmann, M.; Kessler, H.; Geiger, B.; Spatz, J. P. Focal Adhesion Stabilization by Enhanced Integrin-cRGD Binding Affinity. BioNanoMaterials 2017, 18, No. 20160014.

(43) Mas-Moruno, C.; Rechenmacher, F.; Kessler, H. Cilengitide: The First Anti-Angiogenic Small Molecule Drug Candidate. Design, Synthesis and Clinical Evaluation. Anti-Cancer Agents Med. Chem. 2010, 10, 753-768.

(44) Haubner, R.; Finsinger, D.; Kessler, H. Stereoisomeric Peptide Libraries and Peptidomimetics for Designing Selective Inhibitors of the $\alpha \mathrm{v} \beta 3$ Integrin for a New Cancer Therapy. Angew. Chem., Int. Ed. 1997, 36, 1374-1389.

(45) Li, G.; Cao, H.; Zhang, W.; Ding, X.; Yang, G.; Qiao, Y.; Liu, X.; Jiang, X. Enhanced Osseointegration of Hierarchical Micro/ Nanotopographic Titanium Fabricated by Microarc Oxidation and Electrochemical Treatment. ACS Appl. Mater. Interfaces 2016, 8, $3840-3852$ 
(46) Arsiwala, A.; Desai, P.; Patravale, V. Recent Advances in Micro/ Nanoscale Biomedical Implants. J. Control. Release 2014, 189, 25-45. (47) Ding, Y.; Yang, M.; Yang, Z.; Luo, R.; Lu, X.; Huang, N.; Huang, P.; Leng, Y. Cooperative Control of Blood Compatibility and Re-Endothelialization by Immobilized Heparin and Substrate Topography. Acta Biomater. 2015, 15, 150-163.

(48) Nozaki, K.; Shinonaga, T.; Ebe, N.; Horiuchi, N.; Nakamura, M.; Tsutsumi, Y.; Hanawa, T.; Tsukamoto, M.; Yamashita, K.; Nagai, A. Hierarchical Periodic Micro/Nano-Structures on Nitinol and Their Influence on Oriented Endothelialization and Anti-Thrombosis. Mater. Sci. Eng., C 2015, 57, 1-6.

(49) Rechenmacher, F.; Neubauer, S.; Mas-Moruno, C.; Dorfner, P. M.; Polleux, J.; Guasch, J.; Conings, B.; Boyen, H.-G.; Bochen, A.; Sobahi, T. R.; Burgkart, R.; Spatz, J. P.; Fässler, R.; Kessler, H. A Molecular Toolkit to Functionalize Ti-Based Biomaterials That Selectively Control Integrin-Mediated Cell Adhesion. Chem. - Eur. J. 2013, 19, 9218-9223.

(50) Mas-Moruno, C.; Dorfner, P. M.; Manzenrieder, F.; Neubauer, S.; Reuning, U.; Burgkart, R.; Kessler, H. Behavior of Primary Human Osteoblasts on Trimmed and Sandblasted Ti6Al4V Surfaces Functionalized with Integrin $\alpha \mathrm{v} \beta 3$-Selective Cyclic RGD Peptides. J. Biomed. Mater. Res., Part A 2013, 101, 87-97.

(51) Schindelin, J.; Arganda-Carreras, I.; Frise, E.; Kaynig, V.; Longair, M.; Pietzsch, T.; Preibisch, S.; Rueden, C.; Saalfeld, S.; Schmid, B.; Tinevez, J.-Y.; White, D. J.; Hartenstein, V.; Eliceiri, K.; Tomancak, P.; Cardona, A. Fiji: An Open-Source Platform for Biological-Image Analysis. Nat. Methods 2012, 9, 676-682.

(52) Meijering, E.; Dzyubachyk, O.; Smal, I. Methods for Cell and Particle Tracking. Methods Enzymol. 2012, 504, 183-200.

(53) Kemkemer, R.; Jungbauer, S.; Kaufmann, D.; Gruler, H. Cell Orientation by a Microgrooved Substrate Can Be Predicted by Automatic Control Theory. Biophys. J. 2006, 90, 4701-4711.

(54) Maiuri, P.; Rupprecht, J.-F.; Wieser, S.; Ruprecht, V.; Bénichou, O.; Carpi, N.; Coppey, M.; Beco, S. D.; Gov, N.; Heisenberg, C.-P.; Crespo, C. L.; Lautenschlaeger, F.; Berre, M. L.; Lennon-Dumenil, A.M.; Raab, M.; Thiam, H.-R.; Piel, M.; Sixt, M.; Voituriez, R. Actin Flows Mediate a Universal Coupling between Cell Speed and Cell Persistence. Cell 2015, 161, 374-386.

(55) Bozec, L.; van der Heijden, G.; Horton, M. Collagen Fibrils: Nanoscale Ropes. Biophys. J. 2007, 92, 70-75.

(56) Uttayarat, P.; Toworfe, G. K.; Dietrich, F.; Lelkes, P. I.; Composto, R. J. Topographic Guidance of Endothelial Cells on Silicone Surfaces with Micro- to Nanogrooves: Orientation of Actin Filaments and Focal Adhesions. J. Biomed. Mater. Res., Part A 2005, $75,668-680$.

(57) Franco, D.; Klingauf, M.; Bednarzik, M.; Cecchini, M.; Kurtcuoglu, V.; Gobrecht, J.; Poulikakos, D.; Ferrari, A. Control of Initial Endothelial Spreading by Topographic Activation of Focal Adhesion Kinase. Soft Matter 2011, 7, 7313-7324.

(58) Sales, A.; Holle, A. W.; Kemkemer, R. Initial Contact Guidance During Cell Spreading Is Contractility-Independent. Soft Matter 2017, $13,5158-5167$.

(59) Fujita, S.; Ohshima, M.; Iwata, H. Time-Lapse Observation of Cell Alignment on Nanogrooved Patterns. J. R. Soc., Interface 2009, 6, S269-S277.

(60) Goffin, J. M.; Pittet, P.; Csucs, G.; Lussi, J. W.; Meister, J.-J.; Hinz, B. Focal Adhesion Size Controls Tension-Dependent Recruitment of A-Smooth Muscle Actin to Stress Fibers. J. Cell Biol. 2006, 172, 259-268.

(61) Kim, D.-H.; Wirtz, D. Focal Adhesion Size Uniquely Predicts Cell Migration. FASEB J. 2013, 27, 1351-1361.

(62) Kaiser, J.-P.; Reinmann, A.; Bruinink, A. The Effect of Topographic Characteristics on Cell Migration Velocity. Biomaterials 2006, 27, 5230-5241.

(63) Kim, D.-H.; Han, K.; Gupta, K.; Kwon, K. W.; Suh, K.-Y.; Levchenko, A. Mechanosensitivity of Fibroblast Cell Shape and Movement to Anisotropic Substratum Topography Gradients. Biomaterials 2009, 30, 5433-5444.
(64) Dang, I.; Gorelik, R.; Sousa-Blin, C.; Derivery, E.; Guérin, C.; Linkner, J.; Nemethova, M.; Dumortier, J. G.; Giger, F. A.; Chipysheva, T. A.; Ermilova, V. D.; Vacher, S.; Campanacci, V.; Herrada, I.; Planson, A.-G.; Fetics, S.; Henriot, V.; David, V.; Oguievetskaia, K.; Lakisic, G.; Pierre, F.; Steffen, A.; Boyreau, A.; Peyriéras, N.; Rottner, K.; Zinn-Justin, S.; Cherfils, J.; Bièche, I.; Alexandrova, A. Y.; David, N. B.; Small, J. V.; Faix, J.; Blanchoin, L.; Gautreau, A. Inhibitory Signalling to the Arp2/3 Complex Steers Cell Migration. Nature 2013, 503, 281-284.

(65) Saito, A. C.; Matsui, T. S.; Ohishi, T.; Sato, M.; Deguchi, S. Contact Guidance of Smooth Muscle Cells Is Associated with Tension-Mediated Adhesion Maturation. Exp. Cell Res. 2014, 327, 111

(66) Pennisi, C. P.; Dolatshahi-Pirouz, A.; Foss, M.; Chevallier, J.; Fink, T.; Zachar, V.; Besenbacher, F.; Yoshida, K. Nanoscale Topography Reduces Fibroblast Growth, Focal Adhesion Size and Migration-Related Gene Expression on Platinum Surfaces. Colloids Surf., B 2011, 85, 189-197.

(67) González-García, C.; Sousa, S. R.; Moratal, D.; Rico, P.; Salmerón-Sánchez, M. Effect of Nanoscale Topography on Fibronectin Adsorption, Focal Adhesion Size and Matrix Organisation. Colloids Surf., B 2010, 77, 181-190.

(68) Yang, K.; Jung, H.; Lee, H.-R.; Lee, J. S.; Kim, S. R.; Song, K. Y.; Cheong, E.; Bang, J.; Im, S. G.; Cho, S.-W. Multiscale, Hierarchically Patterned Topography for Directing Human Neural Stem Cells into Functional Neurons. ACS Nano 2014, 8, 7809-7822.

(69) Lepzelter, D.; Bates, O.; Zaman, M. Integrin Clustering in Two and Three Dimensions. Langmuir 2012, 28, 5379-5386.

(70) Cukierman, E.; Pankov, R.; Stevens, D. R.; Yamada, K. M. Taking Cell-Matrix Adhesions to the Third Dimension. Science 2001, 294, 1708-1712.

(71) Truong, H.; Danen, E. H. J. Integrin Switching Modulates Adhesion Dynamics and Cell Migration. Cell Adhes. Migr. 2009, 3, 179-181.

(72) Chen, C. S.; Alonso, J. L.; Ostuni, E.; Whitesides, G. M.; Ingber, D. E. Cell Shape Provides Global Control of Focal Adhesion Assembly. Biochem. Biophys. Res. Commun. 2003, 307, 355-361.

(73) Robinson, L.; Hentzell, A.; Robinson, N. D.; Isaksson, J.; Berggren, M. Electrochemical Wettability Switches Gate Aqueous Liquids in Microfluidic Systems. Lab Chip 2006, 6, 1277-1278.

(74) Nishimoto, S.; Bhushan, B. Bioinspired Self-Cleaning Surfaces with Superhydrophobicity, Superoleophobicity, and Superhydrophilicity. RSC Adv. 2013, 3, 671-690.

(75) Yim, E. K. F.; Darling, E. M.; Kulangara, K.; Guilak, F.; Leong, K. W. Nanotopography-Induced Changes in Focal Adhesions, Cytoskeletal Organization, and Mechanical Properties of Human Mesenchymal Stem Cells. Biomaterials 2010, 31, 1299-1306.

(76) Zaman, M. H.; Trapani, L. M.; Sieminski, A. L.; MacKellar, D.; Gong, H.; Kamm, R. D.; Wells, A.; Lauffenburger, D. A.; Matsudaira, P. Migration of Tumor Cells in 3D Matrices Is Governed by Matrix Stiffness Along with Cell-Matrix Adhesion and Proteolysis. Proc. Natl. Acad. Sci. U.S.A 2006, 103, 10889-10894.

(77) Elosegui-Artola, A.; Bazellières, E.; Allen, M. D.; Andreu, I.; Oria, R.; Sunyer, R.; Gomm, J. J.; Marshall, J. F.; Jones, J. L.; Trepat, $\mathrm{X}$; Roca-Cusachs, P. Rigidity Sensing and Adaptation through Regulation of Integrin Types. Nat. Mater. 2014, 13, 631-637.

(78) Chiang, M. Y. M.; Yangben, Y.; Lin, N. J.; Zhong, J. L.; Yang, L. Relationships among Cell Morphology, Intrinsic Cell Stiffness and Cell-Substrate Interactions. Biomaterials 2013, 34, 9754-9762.

(79) Solon, J.; Levental, I.; Sengupta, K.; Georges, P. C.; Janmey, P. A. Fibroblast Adaptation and Stiffness Matching to Soft Elastic Substrates. Biophys. J. 2007, 93, 4453-4461.

(80) Lee-Thedieck, C.; Rauch, N.; Fiammengo, R.; Klein, G.; Spatz, J. P. Impact of Substrate Elasticity on Human Hematopoietic Stem and Progenitor Cell Adhesion and Motility. J. Cell Sci. 2012, 125, $3765-3775$ 